\title{
Sodium Fluorescein Guided Excision of High Grade Gliomas: A Tool for Total Excision
}

\author{
MOHAMED I. REFAAT, M.D. and EHAB A. ABD EL-SALAM, M.D. \\ The Department of Neurosurgery, Faculty of Medicine, Cairo University
}

\begin{abstract}
Background: The best management of high grade gliomas starts with adequate cytoreductive surgery followed by adjuvan therapies. The extent of tumor resection is an important prognostic factor, and it directly affects the tumor progression and the median survival period. Sodium Fluorescein is a fluorescent tracer that crosses the disrupted blood brain barrier, making it suitable for intraoperative visualization of malignant gliomas.
\end{abstract}

Aim: To evaluate the effectiveness of $\mathrm{Na}$ Fluorescein to demarcate tumor tissue from brain tissue.

Material and Methods: This study was conducted on 30 patients who were indicated for surgical excision of radiologically diagnosed high grade gliomas. All cases had pre and immediate post-operative imaging with contrast to detect the amount of tumor excision. In all our cases at the time of opening the dura, after doing sensitivity testing, $15-20 \mathrm{mg} / \mathrm{Kg}$ $\mathrm{Na}$ Fluorescein was administrated intravenously. The visible yellow stained tumor was then excised using the standard microscopic procedure.

Results: All cases had primary brain gliomas (21 were WHO grade IV and 9 cases were grade III). Post-operative imaging showed gross total excision in 23 cases (77\%), near total excision ( $>80 \%$ of tumor removed) in 6 cases $(20 \%)$, and subtotal excision ( $<80 \%$ of tumor removed) in 1 case $(3 \%)$. None of our cases showed any complications related to reaction from Fluorescein, while 2 cases $(6.7 \%)$ had transient post-operative motor weakness, and 1 case (3\%) had postoperative dysphasia.

Conclusion: The use of Sodium Fluorescein has proven to be an effective tool for demarcation of tumoral tissue from brain tissue; this demarcation was evident using the naked eye, as well as the non-filtered white microscopic light. It is a simple, safe technique, and can be used in economically limited centers; in which Neuronavigation isn't available. The use of Fluorescein increases the chances of safe gross total excision of high grade gliomas, which is reflected on increasing the survival rates as well as decreasing the tumor progression.

Key Words: Sodium Fluorescein - High grade glioma - Total excision-Median survival.

Correspondence to: Dr. Mohamed I. Refaat,

The Department of Neurosurgery, Faculty of Medicine, Cairo University

\section{Introduction}

MALIGNANT Gliomas are still the most common primary brain tumors. One and two-year survival rates of patients with high grade gliomas are only $53.7 \%$ and $14.6 \%$ respectively [1] . Treatment of high grade Gliomas remains one the greatest challenges for modern therapies. The best treatment protocol includes an initial aggressive cytoreductive surgical resection, followed chemotherapy and radiation therapy [2]. The extent of tumor resection has been proven to have a direct influence on the prognosis of high grade gliomas and increasing the overall as well as the progression free survival rates. The extent of tumor resection also increases the efficacy of radiation and chemotherapy [3] .

The aim of gross total excision should be to remove all contrast enhancing tissues. This can be limited by the location of the tumor, its proximity to eloquent areas, and the difficulty in identifying the tumor margins due to the infiltrative nature of high grade tumors [4]. The use of the surgical microscope increased the accuracy and efficacy of tumor resection by improving illumination and magnification; however, it still depends on the surgeon's eye to differentiate but tumoral tissue and normal brain parenchyma [2]

Several modern techniques have been developed to facilitate identification of tumor margins and to decrease surgery related morbidities. The techniques included intraoperative neuro-navigation, ultrasonography, and intraoperative MRI [2]. However, the use of these modern techniques might be still limited by its cost and infrastructure requirements [4]. The use of Sodium Fluorescein can be an economically low, yet still an effective tool for differentiating between tumoral tissue and normal brain parenchyma. 
Fluorescence-guided surgery was developed as a tool with the potential to give real time intraoperative visualization for tumor tissue. Stummer et al were the first to publish promising results in the "ALA trial". They published significantly higher extent of tumor resection in the 5-aminolevulinic acid (ALA) group [5]. Fluorescence-guided surgery can be divided into two main lines; endogenous and exogenous fluorescence. Tumor cells will metabolize the orally administrated 5-aminolevulinic acid (ALA), leading to endogenous autofluorescence [6]. Exogenous fluorescence is achieved by IV injection of Sodium Fluorescein, which will cross areas of disrupted blood brain barrier (BBB) to stain the tumoral cells. Sodium Fluorescein can be used in low doses and visualized with special light filters applied to the surgical microscope or in high doses $(20 \mathrm{mg} / \mathrm{Kg}$ body weight) and visualized with regular microscopic white illumination [7]

In our study, we are evaluating the utility of high dose sodium fluorescein-guided surgery with resection of high grade gliomas. We are studying the benefit of using fluorescein in increasing the extent of tumor resection, and possible side effects or complications of this tool.

\section{Patients and Methods}

This is an analytical prospective cohort study that was conducted on cases indicated for surgical excision of radiologically diagnosed high grade gliomas. The targeted sample size was 30 consecutive cases. Patients included in this study were selected to have solitary, supratentorial lesions. Brain stem, thalamic lesions, and intraventricular lesions were not included in our study. Patients with severe renal or hepatic diseases, as well as patients with known hypersensitivity to contrast materials were excluded from this study. The study was conducted between 2014 and 2016 in the Neurosurgery department of Cairo University.

A full clinical history was taken for the cases. Full preoperative and postoperative neurological examination was performed. Routine preoperative laboratory investigations, CT brain and gadolinium enhanced MRI brain were done for all cases preoperatively.

Surgical approach: In all 30 cases standard neurosurgical tumor resection procedure was applied. Non-filtered white light neurosurgical microscope was used in all surgeries. During elevation of the bone flap $5 \mathrm{ml}$ diluted sodium fluorescein in injected intravenously by the anesthetist, and the patient is monitored for 10 minutes for any hyper- sensitivity reaction and for any skin rash. After confirming that no sensitivity reaction occurred full dose of sodium fluorescein $(15-20 \mathrm{mg} / \mathrm{kg}$ of Fluorescein Injection USP 10\% w/v, SUNWAYS (India) PVT.LTD) is then injected intravenously. The visible dark yellow stained tumor was then excised using the standard microscopic procedure. During haemostasis it was taken in consideration to reduce the use of haemostatic agents, as they can be misinterpreted as residual tumor in the follow up images. All cases were operated upon by the same surgical team, to eliminate the bias that could arise from variations of the surgical experience of different surgical teams.

Immediate post-operative clinical and radiological (CT with contrast within 48 hours) follow-up was performed for all cases. The tumor is sent for histopathologic examination.

The data collected from the 30 cases were analyzed for tumors' histopathology, degree of tumor removal, and any postoperative complications. Tumor volumes preoperatively and postoperatively were calculated mathematically from the images (using modified ellipsoid volume calculation formula) by the authors to decrease the bias of the obtained results. According to the extent of tumor removal results were divided to three main categories:

- Gross total excision if $100 \%$ of tumor volume was removed.

- Near total excision if more than $80 \%$ of tumor volume is removed.

- Subtotal excision if less than $80 \%$ of tumor volume is removed.

\section{Results}

Patient population: There were 16 (53\%) females and 14 males (47\%), their age ranged from 26 to 66 years; the mean age in our study was 50.8 years (standard deviation 9.4). All cases included in the study were chosen to have radiologically diagnosed solitary, supratentorial high grade malignant gliomas. Deep basal ganglia lesions, intraventricular lesions, as well as infratentorial lesions were not included in this study.

Clinical picture: The most common presenting symptom among our cases was manifestations of increased intracranial pressure (ICP), in 21 of our 30 cases $(70 \%)$. The duration of symptoms ranged from 1 to 9 days to 2 (mean 3.3 days, SD 4.2). Preoperative Karnofsky Performance scale (KPS) of the cases included in this study ranged from 20 to 90 (mean 74, SD 23). 
Anatomically all tumors included in this study were chosen to be solitary supratentorial, excluding basal ganglia and intraventricular tumors. Tumor volumes ranged from 25 to $115 \mathrm{cc}^{3}$. The mean volume was $60.1 \mathrm{cc}^{3}$.

Standard micro neurosurgical principles were followed during surgical excision of all tumors. All tumors showed dark yellow staining after Sodium Fluorescein injection. The yellow color was observed in 28 cases $(93 \%)$ under the normal white light of the microscope. In 23 of these cases the tumor was stained in dark yellow color (greenish yellow), while in the rest of the tumors, the color was light yellow. Clear demarcation between the tumor edges and the normal parenchyma was achieved in all positively stained cases. The average time for tumor staining was 15 minutes (range 10 to 30min.) and lasted till closure of the dura. (Average tumor resection time was $94 \mathrm{~min}$.) None of our cases had any immediate or delayed reaction from the Sodium Fluorescein. All patients had yellowish staining of urine and sclera. This staining disappeared in an average time of $14 \mathrm{hrs}$. Postoperative liver and kidney functions didn't show any changes related to the use of the Fluorescein dye. Postoperative histopathologic examination revealed: 1- Glioblastoma multiforme WHO GIV in 21 cases (70\%), 2- Anaplastic Astrocytoma WHO GIII in 9 cases $(30 \%)$.

Tumor removal: The extent of tumor removal was determined in each by analysis of the postoperative imaging studies, calculating the percentage of tumor volume left behind. We had gross total excision in 23 cases $(77 \%)$, near total excision in 6 cases $(20 \%)$, and subtotal excision in 1 case $(3 \%)$.

Post-operative course: There were no intraoperative mortalities. 3 of our cases $(10 \%)$ experienced 2 new postoperative neurological findings including: 2 cases $(6.7 \%)$ had transient postoperative motor weakness that resolved after the use of brain dehydration. One case had postoperative dysphasia. Postoperative Karnofsky Performance scale (KPS) of the cases study ranged from 40 to 100 (mean 81, SD 16).

Table (1): Clinical presentations of cases.

\begin{tabular}{lcc}
\hline Clinical manifestation & Number of cases & Percentage \\
\hline Headache and vomiting only & 15 & 50 \\
Headache, vomiting, & 4 & 13.3 \\
$\quad$ and motor weakness & 2 & 6.7 \\
Headache, vomiting, & & \\
$\quad$ motor weakness, and aphasia & 6 & 20 \\
Seizures & 3 & 10 \\
Disturbed conscious level &
\end{tabular}
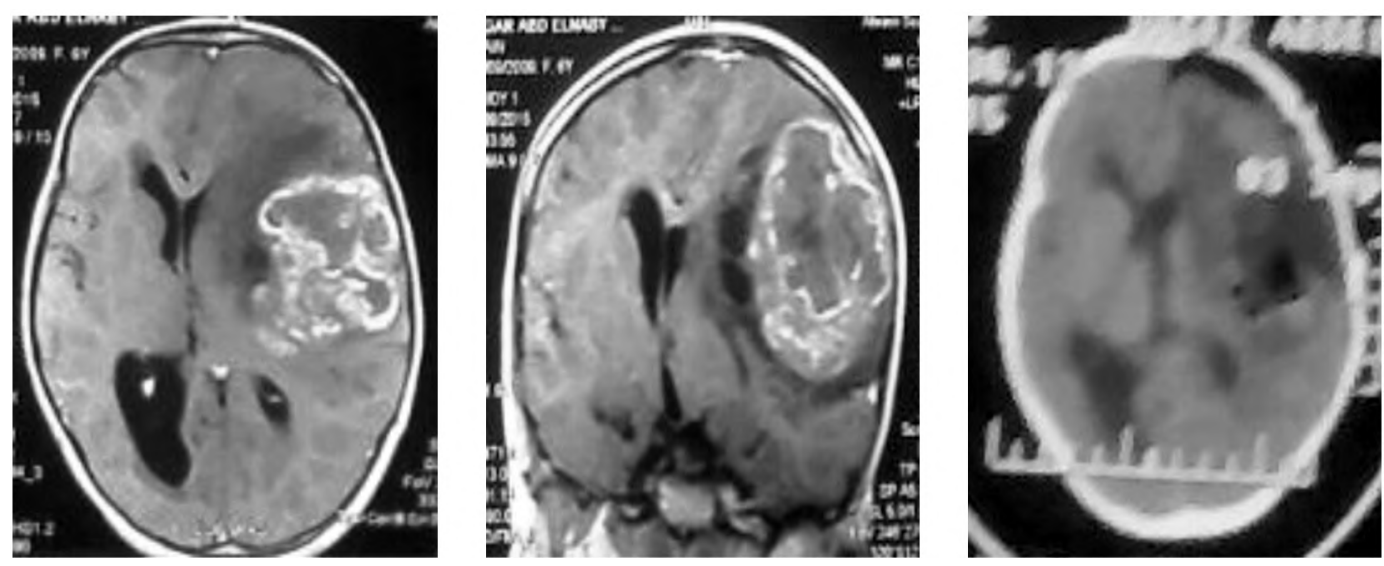

Fig. (1): Preoperative MRI of a 26 years old female, presenting with headache, vomiting, and right sided hemiparesis. Postoperative CT showed gross total excision. Pathology was: Glioblastoma Multiforme. Patient did well with no postoperative complications.
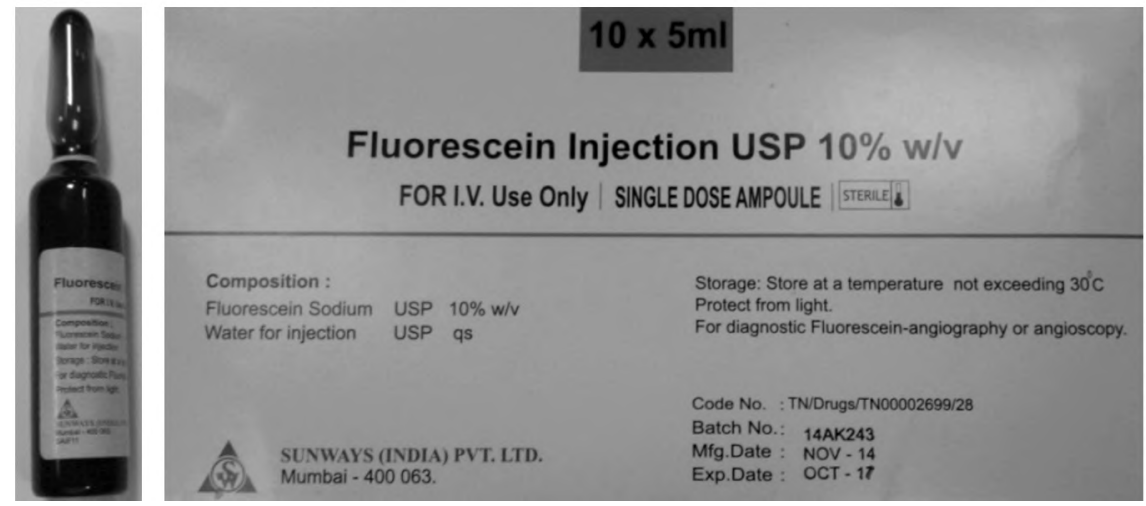

Fig. (2): $15-20 \mathrm{mg} / \mathrm{kg}$ of Fluorescein Sodium Injection USP 10\% w/v, product of SUNWA- YS (India) PVT. LTD was used in our stu-dy. 

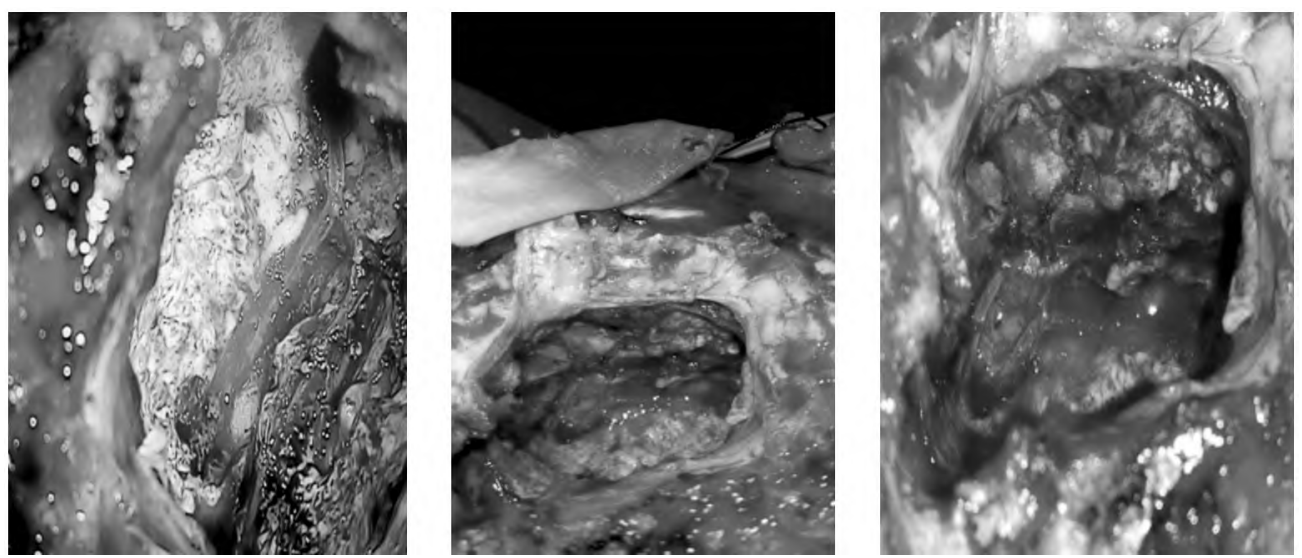

Fig. (3): Intraoperative images showing the greenish yellow staining of the tumor after Fluorescein injection. Tumor staining was clear using both naked eye as well as standard non-filtered microscopic light.
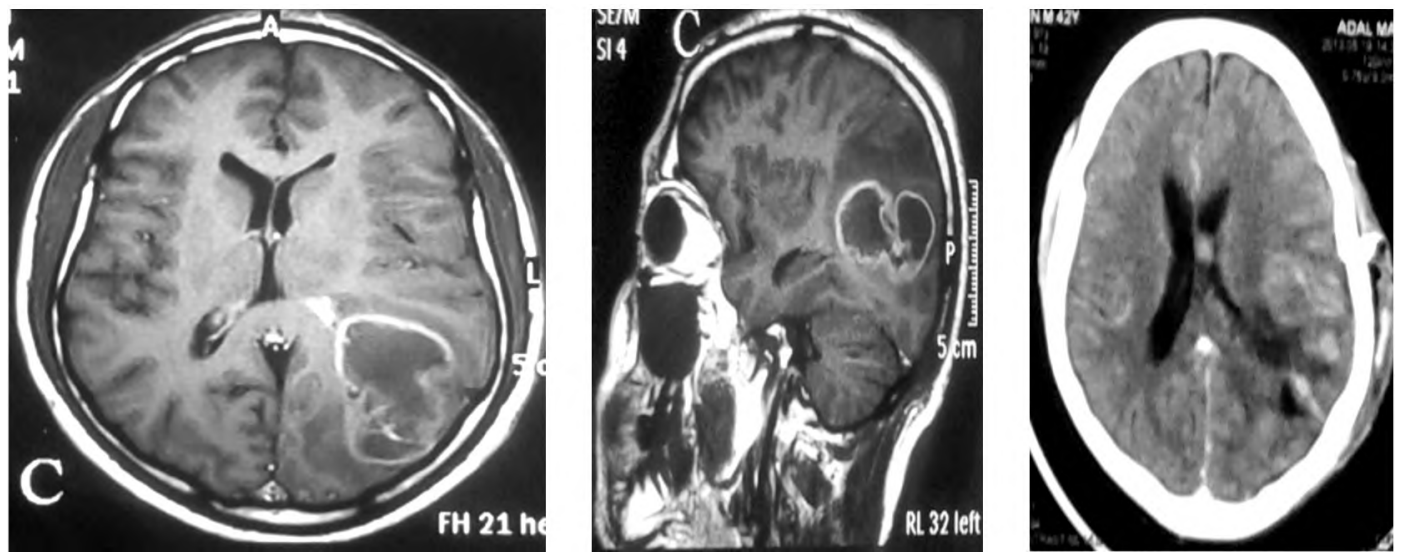

Fig. (4): MRI of a 41 years old male presenting with headache and vomiting. Postoperative CT showing gross total excision of tumor. Pathology was Glioblastoma Multiforme.
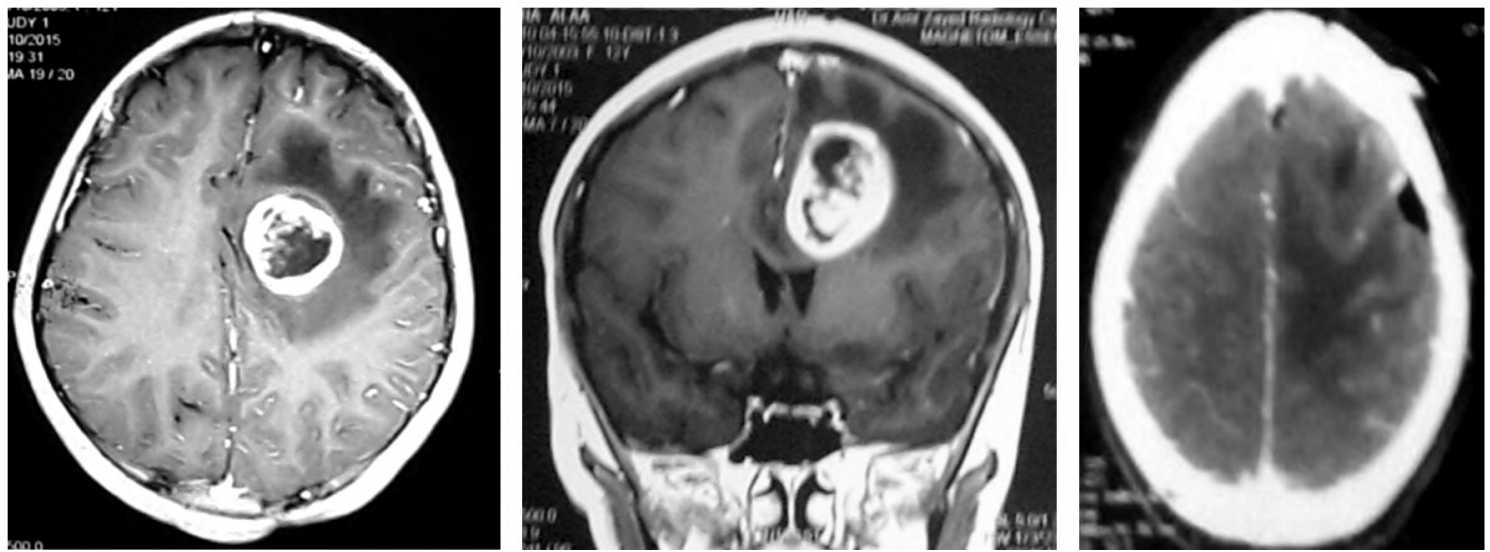

Fig. (5): MRI of a 42 years old male presenting with right sided weakness. Postoperative CT showing gross total excision of tumor. Pathology was Anaplastic astrocytoma GIII.

\section{Discussion}

Gross total resection of malignant gliomas remains as a corner stone in all treatment protocols. There is a growing amount of evidence indicating that it directly affects the outcome as well as the survival rates and the progression free survival periods. Being radical in tumor resection increases the efficacy of postoperative radiation and chemotherapy. The intraoperative differentiation between the tumor margins and the normal parenchyma limits the extent of tumor resection. In the past few decades new techniques are being developed by neurosurgeons to increase the amount of tumor resection and help in identification of tumor margins. These techniques include intraoperative 
MRI, ultrasonography, neuronavigation and. Among these techniques Fluorescein guided resection remains a simple, economically feasible, effective technique of identification of tumoral tissues $[4,8]$

In this study we investigated the efficacy of the use of high dose Sodium Fluorescein injection $(15-20 \mathrm{mg} / \mathrm{kg})$ to differentiate between tumor tissues and normal parenchyma under the normal white light of the surgical microscope, and its influence of the rates of tumor resection.

Our study included 30 cases, their mean age was 52 years, $53 \%$ were females, and $70 \%$ presented with manifestations of increased ICP. The mean duration of symptoms was 10 days, and the mean preoperative KPS score was 60 . The mean tumoral volume was $60.1 \mathrm{cc}^{3}$. This study group was matching with several published studies including the study published by Schebesch KM et al., [9] and the also the study published by Acerbi F et al., [10] .

The use of the dye was found to be helpful in majority of cases, $93.3 \%$ of cases showed variable degrees of yellow staining after injection of the dye. Clear demarcation between the tumor edges and the normal parenchyma could be achieved in all these cases. None of our cases presented any adverse effects to the dye. This was matching the results published by Bo Chen et al., who described three grades of yellowish staining in $90 \%$ of their studied sample [4]. These values were even better than the results published by Schebesch KM et al., [9] who used low dose of sodium fluorescein and a special light filter applied to the microscope, as found the dye helpful in $80 \%$ of their studies cases.

Postoperative histopathologic examination revealed Glioblastoma multiforme WHO GIV in $70 \%$ of cases, and Anaplastic Astrocytoma WHO GIII in $30 \%$. We had gross total excision in $77 \%$ of cases, near total excision in $20 \%$, and subtotal excision in $3 \%$. We had $10 \%$ transient postoperative morbidities and $33 \%$ improvement in the mean postoperative KPS score. Shinoda et al., reported gross total resection in $84.4 \%$ of their 32 cases with the use of high dose Sodium Fluorescein [11] Also, Koc et al., reported statistically significant difference between their two studied groups, as they achieved GTR in $83 \%$ of the Fluorescein group, compared to GTR in $54.5 \%$ of the control group [12]. Also, the study published by Bo Chen et al showed significant differences in the GTR and the progression free survival period between their two groups, in favor using the Fluorescein dye [4].

\section{Conclusion:}

The use of the fluorescent dye Sodium Fluorescein in high dose has proven be a helpful tool for gross total resection of malignant gliomas. The yellowish staining of the tumors is clearly visualized with both naked eye and normal white light surgical microscope. It is a simple, safe technique, and can be used in economically limited centers; in which Neuronavigation isn't available. The use of Fluorescein increases the chances of safe gross total excision of high grade gliomas, which is reflected on increasing the survival rates as well as decreasing the tumor progression.

\section{References}

1- BABU R. and ADAMSON C.: Fluorescence-Guided Malignant Glioma Resections. Curr. Drug. Discov. Technol., 9 (4): 256-67, 2012.

2- MISSIOS S., ABBASSY M., et al.: Use of Image Fluorescence in the Resection of Gliomas. Curr. Surg. Rep., 3: 76,2015 .

3- SCHEBESCH K.M. and BRAWANSKI A.: Fluorescein Sodium-Guided Surgery of Malignant Brain Tumors: History, Current Concepts, and Future Projects. Turk Neurosurg, 26 (2): 185-194, 2016.

4- BO CHEN, WANG H., PENGFEI G., et al.: Gross Total Resection of Glioma with the Intraoperative Fluorescenceguidance of Fluorescein Sodium. Int. J. Med. Sci., 9 (8): 708-7 14, 2012

5- STUMMER W., PICHLMEIER U., MEINEL T., et al.: Fluorescence-guided surgery with aminolevulinic acid for resection of malignant glioma: A randomized controlled multicenter phase III trial. Lancet. Oncol., 7: 392-401, 2006.

6- SENDERS J., MUSKENS I., SCHNOOR R., et al.: Agents for fluorescence-guided glioma surgery: A systematic review of preclinical and clinical results. Acta Neurochir (Wien), 159 (1): 151-167, 2017.

7- ORRINGER D., LAU D., KHATRI S., et al.: Extent of resection in patients with glioblastoma: Limiting factors, perception of resectability, and effect on survival. J. Neurosurg., 117 (5): 851-859, 2012.

8- ACERBI F., CAVALLO C., BROGGI M., et al.: Fluorescein-guided surgery for malignant gliomas: A Review. Neurosurg. Rev., 37 (4): 547-57, 2014.

9- SCHEBESCH K.M., PROESCHOLDT M., HOHNE J., et al.: Sodium fluorescein-guided resection under the yellow 560nm surgical microscope filter in malignant brain tumor surgery-a feasibility study. Acta. Neurochir., 155: 693-699, 2013.

10- ACERBI F., BROGGI M., ECOLI M., et al.: Fluoresceinguided surgery for grade IV gliomas with a dedicated 
filter on the surgical microscope: Preliminary results in 12 cases. Acta. Neurochir. (Wien), 155 (7): 1277-86, 2013

11- SHINODA J., YANO H., YOSHIMURA S., et al.: Fluorescence-guided resection of glioblastoma multiforme by using high-dose fluorescein sodium. Technical note. J. Neurosurg., 99: 597-603, 2003.

12- KOC K., ANIK I., CABUK B., et al.: Fluorescein sodiumguided surgery in glioblastoma multiforme: A prospective evaluation. Br. J. Neurosurg., 22: 99-103, 2008.

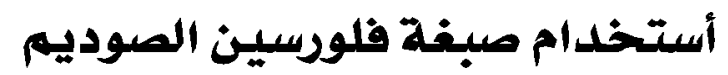

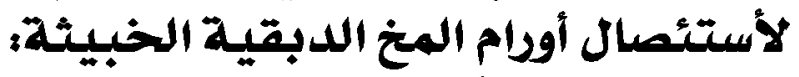

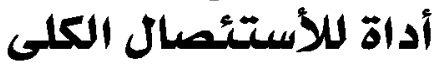

ملخص البحث: أجريت هذه الدراسة على ثلاثقن مريضاً ممن كانوا يعانون من أودام دبقية مخية خبيثة. تم تجميع الصالات ما بين

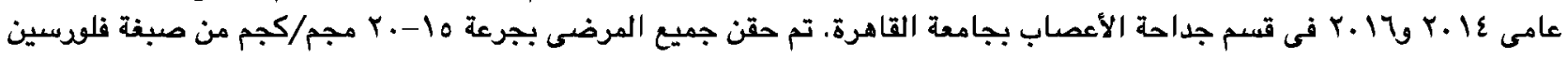

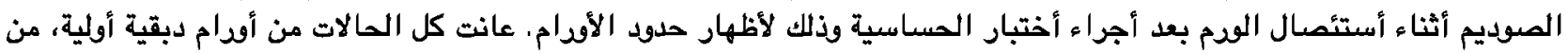

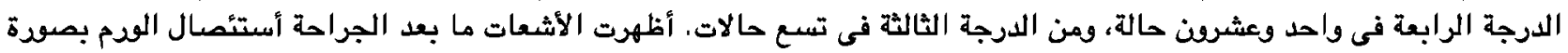

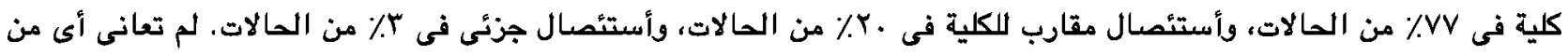

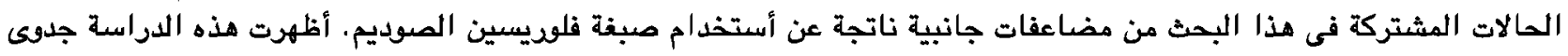

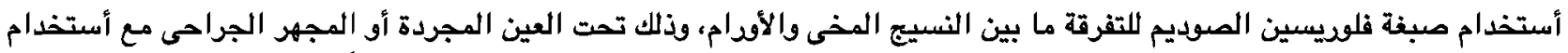

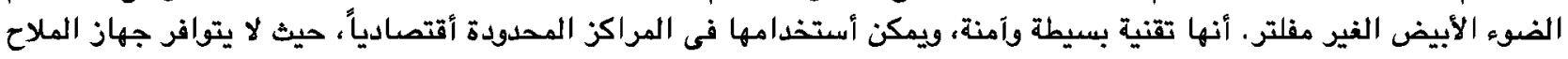

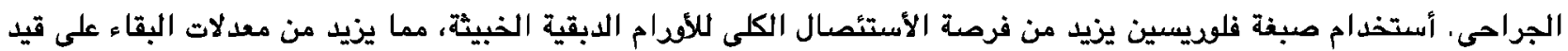

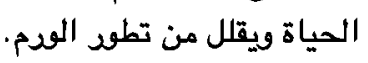

